effectiveness factors. The relation of selectivity to the catalyst size is not so simple as was shown in the case of linear kinetics and a uniform pore model. It was found that from the experimental data and the structure of the catalyst the micro and macro pores model is most reasonable and the experimental data on selectivity coincides with the model by the consecutive reactions of Langmuir-Hinshelwood rate forms.

In general if the rate form is Langmuir type, the selectivity decreases more than it by the linear kinetics and this effect becomes more remarkable by the increase of adsorption constant.

Of course these experimental results might be illustrated by other models or reaction mechanisms. There remains yet some possibility because of the uncertainity on the experimental accuracy. Progress in the study on catalytic processes will analyze them more definitely.

The authors wish to express their sincere thanks to Mr. M. Motoki for his contribution to the preliminary research, and to $\mathrm{Mr}$. K. Adachi of Engineering Research Institute for the measurements with electron microscope.

\section{Nomenclature}

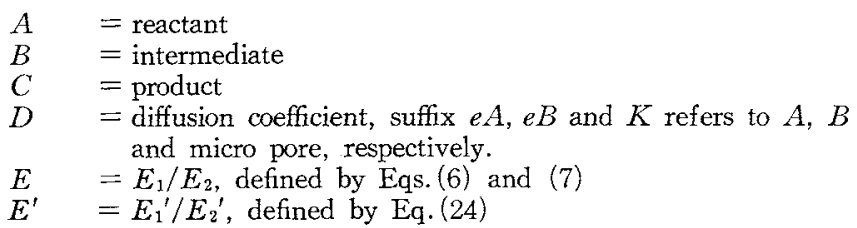

$K_{A} \quad=$ the equilbrium constant of adsorption of acetylene

$k \quad=k_{1}$ and $k_{2}$; the rate constants of the first, and second step reactions: $k^{0}$ refers to the apparent rate constant, and $k^{\prime}$ refers to the rate constant in micro pore

$l=$ the length of the porous matter

$L \quad=$ the radius of the micro particles

$m, m^{\prime}=$ modulus defined by Eqs. (7) and (24), respectively

$p \quad=$ partial pressure, suffix $o$ means the value outside the particle

$p_{A}=$ partial pressure of acetylene

$p_{E} \quad=$ partial pressure of ethylene

$p_{H_{2}}=$ partial pressure of hydrogen

$R \quad=$ the radius of the catalyst particle

$r_{1}, r_{2}=$ catalytic reaction rate of the first and second reaction

$r \quad=$ radial distance in a catalyst particle

$S \quad=$ selectivity, $S^{0}$ refers to it by $p_{B 0}=0$

$S_{p}, S_{m}=$ selectivity in the reactor of piston flow type and of complete mixing type

$s \quad=k_{1} / k_{2}, s^{\prime}$ is equal to $k_{1}^{\prime} / k_{2}^{\prime}$

$X=$ conversion of $A$

\section{Literature cited}

1) Bond, G. C., Catalysis by Metal, Academic Press., London (1962)

2) Bond, G. C., J. Chem. Soc., 2705 (1958)

3) Bond, G. C and Mann, R. S., J. Chem. Soc., 4738 (1958)

4) Butt, J. B., Chem. Eng. Sci., 21, 275 (1966)

5) Carberry, J. J., Chem. Eng. Sci., 17, 675 (1962)

6) Hutchings, J and Carberry, J. J., A. I. Ch. E. Journal, 12, $20(1966)$

7) Sheridan, J., J. Chem. Soc., 133, 301 (1945)

8) Sheridan, J., J. Chem. Soc., 373 (1944)

9) Wakao, H and Otsuka, Y., Annual Meeting, Chem. Eng. Soc. Japan, April (1966)

10) Wheeler, A., Catalysis (Edited by Emmett P. H.) Vol. II Reinhold, New York (1955)

\title{
THE EFFECT OF MICRO-MIXING ON THE HOMOGENEOUS POLYMERIZATION OF STYRENE IN A CONTINUOUS FLOW REACTOR*
}

\author{
MAKOTO HARADA**, KATSUJI TANAKA****, WATARU EGUCHI** AND \\ SHINJI NAGATA*** \\ Engineering Research Institute, Kyoto Univ. Uji
}

\begin{abstract}
Homogeneous polymerization of stysene was carried out in a continuous stirred tank reactor, and it was made clear that special attention should be paid to the segregation of reaction mixture in the reactor for the reactor design of polymerization. Both the effect of micro-mixing on the volume efficiency of reactor and on the properties of polymer and the type of impeller to bring the reaction field homogeneous were discussed.
\end{abstract}

\section{Introduction}

For the fluid mixing which takes place in a continuous

* Received on December 2, 1967

** Engineering Research Institute, Kyoto Univ. (Uji)

*** Dept. of Chemical Engineering, Kyoto Univ. (Kyoto)

**** Teijin Co., Ldt. (Iwakuni) flow reactor, two different mechanisms must be considered. The one is macro-mixing resulting from the residence time distribution of fluid elements in the reactor and the other is micro-mixing caused by the mass exchange between the fluid elements in the reactor.

Danckwerts ${ }^{2)}$ and $Z$ wietering ${ }^{12)}$ introduced the concept 


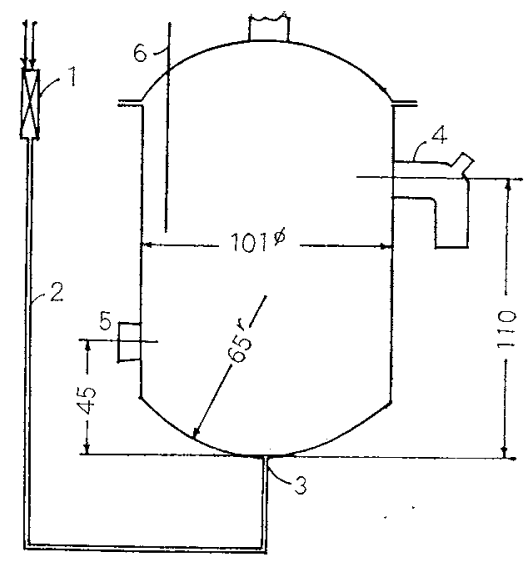

a) reactor vessel
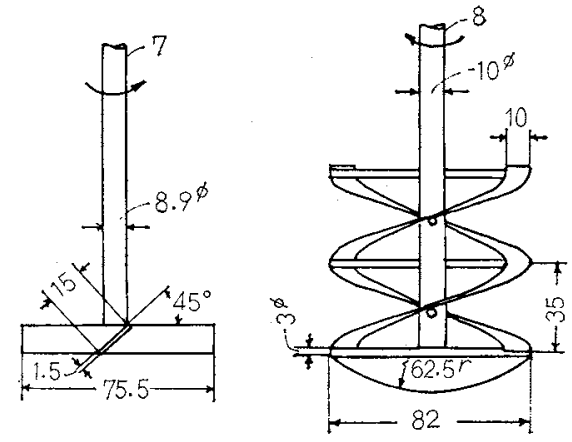

b) impeller
1 premixer

2 capiller

3 inlet of reactor

4 outlet of reactor

Fig. 1

5 side inlet of dextrin

6 thermocouple or thermometer

7 paddle impeller

8 ribbon spiral impeller

Experimental apparatus of the intensity of segregation for the micro-mixing and made theoretical discussions on the characteristics of the continuous flow reactor. The effect of micro-mixing, i. e. coalesence and redispersion of the dispersed droplets on the heterogeneous reaction has been discussed by several investigators ${ }^{1,3,9,10)}$ and the importance of this effect in the analysis of the reactor has been pointed out experimentally ${ }^{6 \sim 8,11}$. In the homogeneous reaction, the rate of mass exchange between fluid elements seems to be very high and thus the effect of mass exchange on the reactor performance has been ignored. However, it may be deduced that the micro-mixing becomes one of the important factors for the continuous reactor performance, when the fast reaction and the reaction in the field of higher viscosity are considered. For the polymerization reaction which took place in the reaction field of relatively lower viscosity, the validity of the assumption of the perfect micro-mixing was confirmed in the previous paper ${ }^{3}$. In this paper, the effect of micro-mixing on both the conversion of polymerization and the average degree of polymerization will be discussed for the case that the polymerization proceeds in the viscous reaction field.

\section{Experimental Apparatus and Procedure}

Polymerization of styrene in toluene using $\alpha, \alpha^{\prime}$-azobisisobutyronitril (ABIN) as initiator was performed in a continuous stirred tank reactor. The materials used were purified by the same way as in the previous paper ${ }^{4}$.

Polymerization reactions were carried out in the stirred tank reactor shown in Fig. 1 which was a cylindricalglass vessel equipped with the impeller made of stainless steel. Two types of impellers, paddle and spiral shown in Fig. $1 \mathrm{~b}$ were used.

Styrene monomer and ABIN dissolved in toluene were fed separately, from their reservoirs in to the premixer with small volume at the desired constant flow rates, and then the mixture was introduced into the bottom of the reactor. The pressure of the reservoirs were kept constant with deoxygenized nitrogen to assure con. stant flow rate. The reaction mixture was removed from the reactor by overflow through the exit pipe placed at the side wall of the reactor. Reaction mixture was sampled out of the effluent stream. The conversion of styrene and the average degree of polymerization were measured by the same method as used in the previous paper ${ }^{4}$. The concentrations of monomer and $A B I N$ in the premixer were adjusted to $6.90 \mathrm{~g}$ moles/liter and $0.0342 \mathrm{~g}$-moles/liter, respectively. The reaction was allowed to proceed in nitrogen atomosphere and at $80.0^{\circ} \mathrm{C}$.

In any run, the temperature difference between the reaction mixture at the reactor wall and the center of the reactor was less than $2^{\circ} \mathrm{C}$. The impeller speed was varied in the range from 180 to $250 \mathrm{r} . \mathrm{p} . \mathrm{m}$. for the paddle impeller and from 80 to 150 r.p.m. for the rib. bon spiral impeller.

Prior to the experiments of reaction, the measurement of residence time distribution was carried out with the use of dextrin solution as the viscous liquid and sodium chloride aqueous solution as the tracer. The concentration of sodium chloride was measured by titration method.

\section{Experimental Results and Discussion}

\section{Macro-mixing of the reactor}

To identify the characteristics of micro-mixing of the reactor used, step responce method was used. Fig. 2 shows the experimental results of $1-F(t)$. In these experiments, the viscosity of the mixture of the dextrin solution and sodium chloride solution was 35 poise and the mean residence time was $120 \mathrm{~min}$. The points shown by symbols $\bigcirc$ and $X$ represent the experimental results on the paddle and ribbon spiral impellers, respectively. The experimental results fall in a straight line with slope -1 through the origin and, therefore, it can be said that the mixture in the reactor is in a perfectly macro-mixed state, i. e. statistically perfect mixing state. Since the value of viscosity, 35 poise approximately corresponded to the one of the reaction mixture at the conversion of 0.75 and further the reaction mixture of the polymerization was ascertained to 
show Newtonian fluid behavior approximately, the reaction mixture might be viewed as a perfectly macromixed state when the reactor was operated at the condition of mean residence time more than 120 min and of viscosity less than 35 poise.

Conversion of styrene and average degree of polymerization

Let the case be considered that the polymerization reaction proceeds at a steady state in the continuous flow reactor in which the reaction mixture is in a perfectly macro-mixed state.

The conversion of styrene and the average degree of polymerization may be estimated from the values computed for the ultimate two cases of degree of micromixing, no micro-mixing and perfect micro-mixing, using the rate equations for elementary reactions developed in the previous paper ${ }^{4)}$.

1) Case of no micro-mixing reactor:

In this case, the conversion of styrene $f_{M, N}$ is given as follows,

$$
f_{M, N}=\int_{0}^{\infty} f_{M, B} \exp (-t / \theta) d(t / \theta)
$$

where, $\theta$ represents the mean residence time and $f_{M, B}$ denotes the conversion of styrene obtained at the reaction time, $t$ by batch operation under the same reaction conditions as the flow operation. The curve $b$ in Fig. 3 shows the value of $f_{M, N}$ calculated by the Eq. (1) with the value of $f_{X, B}$ shown by the curve a in Fig. 3.

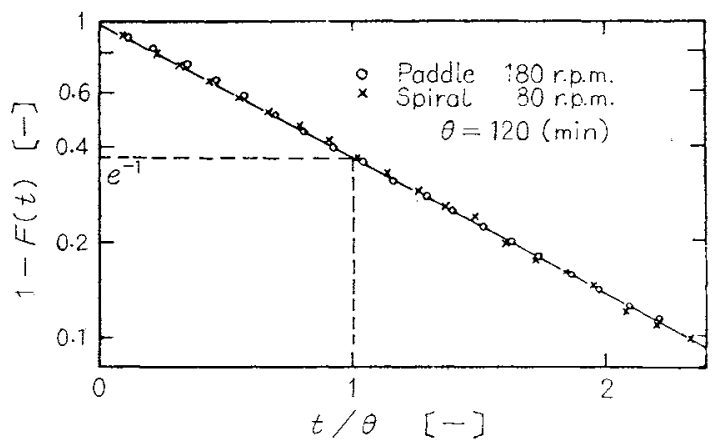

Fig. 2 Validity of the assumption of perfectly macro-mixed reactor
The quantities of dead polymer containing $P$ units $n_{P, N}$ obtained by this type of reactor is given by the following equation.

$$
n_{P, N}=\int_{0}^{\infty} n_{P, B} \exp (-t / \theta) d(t / \theta)
$$

where, the value $n_{P, B}$ denotes the quantities of dead polymer containing $P$ units obtained by the batch operation at the reaction time $t$ under the same reaction conditions as those of the flow operation. The viscosity average degree of polymerization of polymer obtained by the batch operation $\bar{P}_{\mu, B}$ and the one obtained by the continuous flow operation $\bar{P}_{\mu, N}$ are given by the following Eqs. (3) and (4) respectively.

$$
\begin{aligned}
& \bar{P}_{\mu, B}^{\alpha}=\sum_{P=2}^{\infty} P^{1+\alpha} n_{P, B} / \sum_{P=2}^{\infty} P n_{P, B} \\
& \bar{P}_{\mu, N}^{\alpha}=\sum_{P=2}^{\infty} P^{1+\alpha} n_{P, N} / \sum_{P=2}^{\infty} P n_{P, N}
\end{aligned}
$$

where, $\alpha$ is the power number of Mark-Howinks' equation. From Eqs. (2), (3) and (4), $\bar{P}_{\mu, N}$ is obtained by the following equation.

$$
\bar{P}_{\mu, N}^{\alpha}=\int_{0}^{\infty} \bar{P}_{\mu, B}^{\alpha} f_{M, B} \exp (-t / \theta) d(t / \theta) / f_{M, N}
$$

The symbol, $\bigcirc$ in Fig. 4 shows the experimental values obtained by the batch operation and the curve $a$ is the smoothed value connecting the observed values. The value $\overrightarrow{\boldsymbol{P}}_{\mu, N}$ calculated by Eq. (5) with the smoothed curve $a$ is shown in Fig. 4 as the curve $b$.

2) Case of perfect micro-mixing reactor, i. e. ideally mixed reactor :

Since the concentrations of monomer and the others are uniform throughout the perfect micro-mixing reactor ${ }^{*}$ the conversion of monomer is expressed as the following equation with the mass balance.

$$
1-f_{M, P}=\frac{1}{1+(\gamma \delta)^{1 / 2}\left(d f_{M, B} / d t\right)_{i}\left(1-f_{K, P}\right)^{1 / 2} \theta}
$$

where,

$$
\left(d f_{M, B} / d t\right)_{i}=\left(k_{p}{ }^{2} \phi / k_{t}\right)^{1 / 2}\left(2 k_{d} C_{K, i}\right)^{1 / 2}
$$

and $k_{p}, k_{t}$ and $k_{d}$ represent the reaction rate constants of propagation, termination and initiator decomposition, respectively. The values $\psi$ and $f_{K, P}$ are initiator efficiency and conversion of initiator, respectively. As mentioned in the previous paper ${ }^{4,5}$, the value of $(\gamma \delta)$

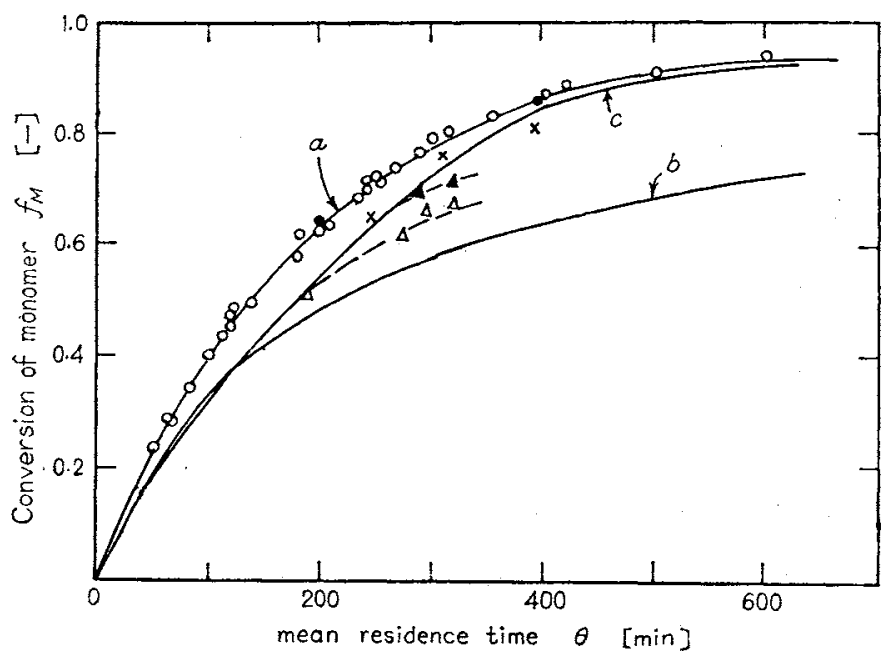

$\begin{array}{cllc}\text { key } & \text { Type } & \text { Impeller } & \text { speed } \\ \bigcirc & \text { Batch } & \text { Paddle } & 150 \text { r.p.m. } \\ \triangle & \text { Batch } & \text { (Ample) } & 0 \\ \triangle & \text { Flow } & \text { Paddle } & 180 \\ \triangle & \text { Flow } & \text { Paddle } & 250 \\ \times & \text { Flow } & \text { Spiral } & 80 \sim 150\end{array}$

curve a; Batch

curve $b$; No micro-mixing (flow) curve $c$; Ideal mixing (flow)

Fig. 3 Conversion of styrene 


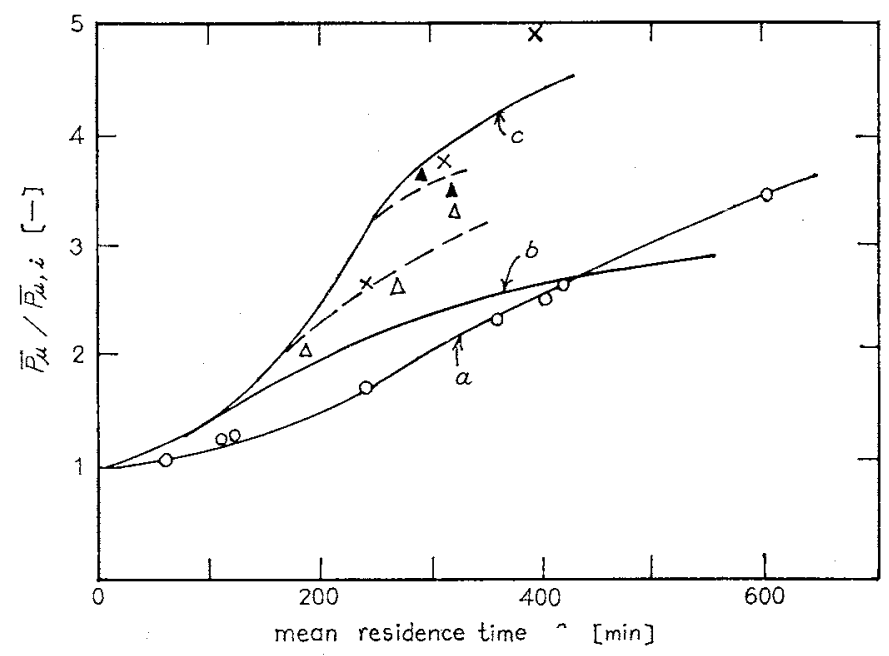

$\begin{array}{cccc}\text { key } & \text { Type } & \text { Impeller } & \text { speed } \\ \bigcirc & \text { Batch } & \text { Paddle } & 150 \text { r.p.m. } \\ \triangle & \text { Flow } & \text { Paddle } & 180 \\ \times & \text { Flow } & \text { Paddle } & 250 \\ \times & \text { Flow } & \text { Spiral } & 80 \sim 150 \\ \text { curve a; } \text { Batch } & & \\ \text { curve b; No micro-mixing (flow) } & \text { No } \\ \text { curve c; Ideal mixing (flow) }\end{array}$

Fig. 4 Viscosity average degree of polymerization

obtained by the batch reactor can be determined apparently by the polymer weight fraction in the reaction mixture, independently of the reaction conditions, i. e. initial concentrations of monomer and initiator and the reaction temparature, and thus it may be also used in the flow operation. The curve $c$ in Fig. 3 was calculated by Eq. (6) using the data in the previous pa$\operatorname{per}^{4)}$.

The average degree of polymerization can be obtained as follows,

$$
\bar{P}_{\mu, H} / \vec{P}_{\mu, i}=(\gamma \delta)^{1 / 2}\left(1-f_{M, P}\right) / \varepsilon\left(1-f_{K, P}\right)^{1 / 2}
$$

where, $\varepsilon$ represents the ratio of initiator efficiency at conversion $f_{M, P}$ to the one at $f_{M, P}=0$ and is also determined only by the polymer weight fraction in the reaction mixture ${ }^{4}$. The curve $c$ in Fig. 4 was calculated by Eq. (8) with the values $(\gamma \delta), \varepsilon, f_{M, P}$ and $f_{K, P}$.

Generally, in the isothermal operation of the reaction with the reaction order higher than unity, the conversion obtained by the perfectly macro-mixed reactor is lower than that obtained by the plug flow reactor or batch operation, and the conversion obtained decreases with the increase in the degree of micro-mixing. The reaction rate of styrene polymerization initiated by $A B I N$ can be described as being of first order in styrene and a half order in ABIN. In the range of lower converison where auto-accerelation effect may be neglected, the conversion obtained by batch operation is the highest. The conversion given by the curve $b$ should be higher than the one of the curve $c$, theoretically. With the increase in the extent of the polymerization reaction, the auto-accerelation effect becomes appreciable and can not be neglected. In the plug flow reactor or batch operation, this effect gradually appears with the increase in the extent of reaction. While, in an ideally mixed reactor, the feed stream is dispersed immediately in the

* It is generally accepted that the auto-accerelation effect expressed by the term $\delta$ in Eq. (6) originates from the diffusion controlled termination reaction. In this case, it is doubtful that a state of perfect mixing in molecular scale exists in principle. The state of perfect micro-mixing defined in this paper denotes a state that the concentrations of several substances are uniform throughout the reactor over the distance equal to the scale of radical segregation caused by the diffusion controlled termination. circumstance where the auto-accerelation effect is remarkable, and thus the apparent reaction rate coefficient of polymerization markedly increases throughout the reactor. Therefore, the conversion obtained by an ideally mixed reactor increases steeply with the increase in the value $\theta$ or $f_{M}$ beyond the conversion of 0.4 and approaches to the value given by the curve $a$ in the range of higher conversion under the operating condition of this experiment. While, the conversion obtained by a no micro-mixing reactor is less sensitive for the autoaccerelation effect and the conversion given by the curve $b$ becomes lower than the curve $c$ in the range where the auto-accerelation effect can not be neglected.

For the average degree of polymerization, the curve $c$ computed on the assumption of an ideally mixed reactor is the highest in the range of higher conversion, because the average degree of polymerization is more sensitive for the auto-accerelation effect than the conversion ${ }^{4}$.

The experimental results of flow system are shown in Figs. 3 and 4 . The points shown by the symbols $\triangle$ and $\boldsymbol{\Delta}$ show the results obtained with the paddle impeller at the stirrer speed 180 and 250 r. p. m., respectively. In comparatively low conversion, the experimental results agree with the value which are calculated on the assumption of the perfectly micro-mixed reactor. As the mean residence time and the conversion of styrene increase, the experimental results deviate from the curve $c$ and approach to the curve $b$. The points shown by the symbols $x$ in Fig. 3 and 4 indicate the experimental results obtained by the reactor with the ribbon spiral impeller and fall in the curve $c$ approximately.

Let us consider the reason why the experimental values do not fall in the curve $c$ calculated on the assumption of an ideally mixed state. For the polymerization reaction, there are several special characters caused by the complexity of reaction: mechanisms and remarkable increase in the viscosity of reaction mixture with the increase in the extent of the reaction. The polymerization reaction seems to be independent of stirrer speed since, as clearly. seen from Fig. 3, the results obtained by ample tube with the diameter $3 \mathrm{~mm}$ agree well with 
those obtained by the batch reactor maintaining the paddle impeller speed 150 r.p. m.. Though some errors may be associated with the determination of conversion, these errors seems to be comparatively small and are not sufficient to explain the deviation of experimental results from the curve $c$.

Let us consider the behavior of the inflowing stream in the reactor with the paddle impeller. Under the operating condition of this work, the viscosity of the reaction mixture became comparatively high in the range of higher conversion. The feed stream coming in to the reactor from the bottom was transported upwards in the direction of the paddle impeller in the state segregated from the surrounding reaction mixture and was breaked up into many comparatively coarse streaks by the shearing force near the impeller. The coarse streaks circulated throughout the reactor with the discharged flow from the impeller and was transformed into finner streaks gradually. Finnaly, a crowd of these streaks was carried out of the reactor. It was clarified by the tracer responce that the reactor could be treated as a perfectly macro-mixed reactor and this fact may be caused by the higher circulating rate, i. e. that a circulating time of the discharged flow is short enough compared with the mean residence time. While, the fact, accertained by the inspection of reaction mixture, that the mixture consisted of a great number of fine streaks, shows perhaps that the rate of mass exchange between the streaks was comparatively low.

The deviations of experimental results in Figs. 3 and 4 from curves $c$ is perhaps owing to the lower rate of mass exchange between the streaks.

When the paddle impeller was used, the critical conversion at which experimental point began to deviate from the curve $c$ increased with the increase in the impeller speed.

It is easily seen in the case of constant viscosity that the reaction mixture in the reactor having larger mean residence time should be essencially more homogeneous because the greater degree of micro-mixing might be achieved. In the polymerization reactions, however, the increase in the mean residence time leads to the increase in the viscosity of the reaction field. Therefore, assuming the greater effect of viscosity than that of mean residence time on the degree of micromixing, it is clearly understood that the degree of micro-mixing decreases with the increase in the mean residence time as is observed in the experiments.

The experimental results obtained by the ribbon spiral impeller fall in the curve $c$. The shearing force in the reactor with a paddle impeller is especially strong near the impeller, while in the reactor with a ribbon spiral impeller, convectional circulation and shearing force are distributed uniformly all over the reactor. It is considered that these uniformly distributed circulation flow and shearing force bring on a great advance in the rate of mass exchange between the streaks.

\section{To Conclude}

The polymerization of sytrene was carried out in a continuous stirred tank reactor. The experimental re- sults obtained by the reactor with the paddle impeller did not agree with the value calculated on the assumption of the ideal mixing. The fact mentioned above was explained by the cause that the rate of mass exchange between fluid elements was comparatively low and the content in the reactor was not in the state of the ideal mixing.

It was shown that the effect of micro-mixing on both the volume efficiency of a reactor and the properties of polymer produced was too great to be ignored when autoaccerelation effect was polymerization was not negligible, and the type of impeller to bring the reaction field to an ideally mixed state was discussed.

The mechanisms of micro-mixing and the scale of segregation must be made clear for the research of mixing performance in future.

\section{Nomenclature}

$C_{K}=$ concentration of initiator

$F(t)=$ responce to a step tracer input

$f_{K}=$ conversion of initiator

$f_{M}=$ conversion of monomer

$k_{p}=$ rate constant of propagation reaction [liter/g-moles $\left.\cdot \mathrm{sec}\right]$

$k_{d}=$ rate constant of initiator decomposition reaction $[1 / \mathrm{sec}]$

$k_{t}=$ rate constant of termination reaction [liter/g-moles $\left.\cdot \mathrm{sec}\right]$

$P \quad=$ degree of polymerization

$P_{\mu} \quad=$ viscosity average degree of polymerization

$t=$ time

$\alpha=$ Mark-Howinks power number

$\gamma=$ ratio of density of reaction mixture to the one in feed state

$\delta \quad=\left(k_{p}^{2} \phi / k_{t}\right) /\left(k_{p}^{2} \phi / k_{t}\right)_{i}$

$=\phi / \phi_{i}$

$=$ initiator efficiency

$=$ mean residence time

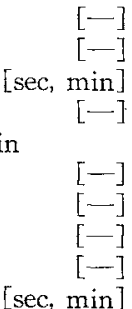

Subscript

$\mathrm{i}=$ state of initial or feed

$\mathrm{B}=$ batch operation

$\mathrm{N}=$ no micro-mixing state

$\mathrm{P} \quad=$ perfect micro-mixing state

\section{Literature cited}

1) Curl, R. N., A. I. Ch. E. Journal, 9, 175 (1963)

2) Danckwerts, P. V., Chem. Eng. Sci., 8, 93 (1958)

3) Harada, M, Arima, K., Eguchi, W., Nagata, S., Mem. Fac. Eng. Kyoto Univ., 24, 431 (1962)

4) Harada, M, Yamada, T., Tanaka, K., Eguchi, W., Nagata, S., Kagaku Kōgaku (Chem. Eng., Japan), 29, 301 (1965), Kagaku Kögaku (Abridged edition), 3, 227 (1965)

5) Harada, M, Yamada, T., Eguchi, W., Nagata, S., Kagaku Kōgaku(Chem. Eng., Japan), 29, 500 (1965), Kagaku Kōgaku (Abridged edition), 4, 1 (1966)

6) Madden, A. J., Damerell, G. L., A. I. Ch. E. Journal, 8, 233 (1962)

7) Matsuzawa, H., Miyauchi, T., Kagaku Kōgaku (Chem. Eng., Japan), 25, 583 (1961)

8) Miller, R. S., Ralph, J. L., Curl, R. L., Towell, G. D., A. I. Ch. E. Journal, 9, 196 (1963)

9) Spielman, L. A., Levenspiel, O., Chem. Eng. Sci., 20, 247 (1965)

10) Rietema, K., Chem. Eng. Sci., 8, 103 (1958)

11) Shinner, R., Church, J. M., Ind. Eng. Chem., 52, 254 (1960)

12) Zwietering, T. N., Chem. Eng. Sci., 11, 1 (1959) 